\title{
Trabajo por proyectos en diseño de maquinaria
}

Rafael R. Sola-Guirado ${ }^{\mathrm{a}}$; Gregorio Blanco-Roldan ${ }^{\mathrm{a}}$; Jesús Gil-Ribes ${ }^{\mathrm{a}}$; Juan AgüeraVega $^{a}$

a E.T.S.I.A.M. Universidad de Córdoba. Ed. Leonardo Da Vinci, Ctra. N IV, km. 396. 14014. Córdoba.

\begin{abstract}
A project-based exercise has been introduced in the subject "Design of agricultural machinery". The students have to go through all phases of product development methodologies: information search, work planning, design, calculation, manufacturing, etc. to solve the problem. At the end, they must obtain a mock-up that emulates the work of an agricultural machine with loader functions. The students chose a methodology of group work by sequential engineering, instead of the one recommended by concurrent engineering, and at the end of the exercise they explained the problems encountered having learned of the pros and cons of both. The exercise covers a practical and enjoyable part of the curricular competences and allows the knowledge of specific engineering software. The practice indicators show the improvement of the teaching quality with greater participation of the students and with better academic results. However, teachers are required an extra dedication, as well as more resources, so in the future the practice should be coordinated with other similar subjects or with continuing education courses.
\end{abstract}

Keywords: mock-up; project; design; learning; machinery

\section{Resumen}

Se propone la introducción de un ejercicio basado en proyectos en la asignatura de "Diseño de maquinaria agraria". Para resolver el problema los alumnos han de pasar por todas las fases de las metodologías de desarrollo de productos: búsqueda de información, planificación del trabajo, diseño, cálculo, fabricación, etc. Al final, deberán obtener una maqueta que modele el trabajo de una máquina agrícola con funciones de cargadora. Los alumnos eligieron una metodología de trabajo en grupo por ingeniería secuencial, en lugar de la aconsejada por ingeniería concurrente, y al final del ejercicio expusieron los problemas encontrados habiendo aprendido de los pros y contras de ambas. El ejercicio cubre de forma práctica y amena gran parte de las competencias curriculares y permite el conocimiento de software específico ingenieril. Los indicadores finales de la práctica muestran la mejora de la calidad docente con mayor participación del alumnado y con mejores resultados académicos. Sin embargo, se requiere del profesorado una dedicación y tiempo extra en la asignatura, así como mayores recursos, por lo que en el futuro se debería intentar la coordinación de la práctica con otras materias similares o con cursos de formación permanente.

Palabras clave: maqueta, proyectos, diseño, aprendizaje, maquinaria 


\section{Introducción}

En el estudio de ingeniería, el método tradicional de resolución de problemas permite poner en práctica los contenidos estudiados en clase. Sin embargo, el "trabajo por proyectos" abre un mayor número de posibilidades, permitiendo a los alumnos afrontar las adversidades que se le plantean, generar hipótesis y tomar decisiones (Mills y Treagust, 2003). Además, acerca al alumno a una realidad más profesional, compleja y global (Hmelo-Silver, 2004).

Un correcto diseño de una práctica basada en proyectos debe aunar diferentes elementos (Han y Bhattacharya, 2001), para abarcar e integrar gran parte de los contenidos cursados, y difícilmente puede obviar la puesta en práctica de otras habilidades adquiridas en otras asignaturas a lo largo de la titulación. Para que el alumnado pueda adquirir las competencias exigidas en el marco laboral, es necesario planificar las actividades con una perspectiva unificada e interdisciplinar (Parra et al., 2011).

En métodos basados en "trabajo por proyectos", el profesor debe dar el control a los propios estudiantes para cambiar su rol tradicional y convertirlos en sujetos activos de construcción y gestión de su conocimiento (Fosnot, 1996). Todo ello, ha de apoyarse en una cooperación entre los estudiantes en la que formen equipos activos de trabajo (García, 2015). Es el grupo el que decide cómo realizar la tarea, qué procedimientos adoptar, cómo dividir el trabajo, y las tareas a realizar. Así, se pretende mantener a los estudiantes comprometidos y motivados en su propio proceso de aprendizaje, lo que posibilita el alcance de sus logros.

Otro aspecto relevante en el curso de materiales ingenieriles, es la necesidad de la utilización de herramientas informáticas que faciliten la resolución de determinadas tareas. Por ello, es esencial que el alumnado conozca y maneje software específico que le permitan adquirir ciertas habilidades para el posterior desempeño profesional (Moursund, 1999).

La asignatura de "Diseño de maquinaria agraria. Automática agraria” ( $4^{\circ}$ curso, Obligatoria, Itinerario de Ingeniería Rural, Grado de Ingeniería Agroalimentaria y del Medio Rural) ha sufrido una reducción del tiempo disponible para la impartición de materia enmarcada en el campo de "Desarrollo de productos". Sin embargo, la evolución de la titulación y el itinerario exige conocer ciertos contenidos como elementos mecánicos, oleohidráulicos, electrónicos y de control. Por tanto, dada la necesidad y la limitación temporal, la mejor forma de hacerlo, es mediante la combinación teórico-práctica, mediante el trabajo en base a proyectos.

\section{Objetivos}

En este trabajo se plantea una práctica docente basada en la resolución de un problema que desemboca en la construcción de un prototipo de máquina agrícola. Para afrontar el trabajo, los alumnos tuvieron que desempeñar una serie de actividades que se encuentran entre las comunes en fases de diseño y desarrollo de productos (DDP), cuyo fundamento teórico se cursó en la asignatura. Se utilizó un enfoque constructivista en el tratamiento interdisciplinar de las actividades planteadas. Para ello, se dotó al alumnado de material de apoyo, como herramientas metrológicas y de taller, y de software específico.

(cc) EY-NC-ND 2018, Universitat Politècnica de València

Congreso In-Red (2018) 
El objetivo general persiguió que el alumnado aplicara los conocimientos adquiridos en la asignatura "Diseño de maquinaria agraria. Automática agraria”, mediante la resolución del problema práctico, planteado como un Proyecto, y en un proceso de colaboración con varios compañeros. Además, se pretendió mejorar la calidad de la asignatura en su ámbito docente. A continuación, se analizan las bases de la práctica planteada.

\section{Desarrollo de la innovación}

PROYECTO: Planificación, diseño, fabricación y automatización de un prototipo que simule una máquina con funciones de manipulación (Figura 1) (cargador frontal, manipuladora telescópica, etc.). Estas máquinas son muy empleadas en el sector agrícola, disponiendo de una conjunción de sistemas mecánicos, oleohidráulicos y eléctricos, lo que justifica su elección para el objetivo del trabajo.

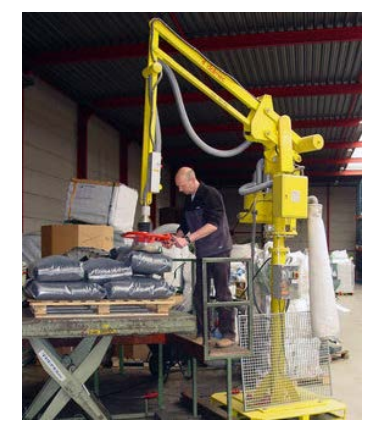

Fig. 1. Máquina cargadora de productos agrícolas.

PROBLEMA: El prototipo (maqueta) no podrá superar las dimensiones de 40x40x40 cm ni el peso de $2 \mathrm{~kg}$ y deberá trasladar un objeto de peso y dimensiones determinadas (paquete de pañuelos) desde un punto inicial hasta otro situado a $10 \mathrm{~cm}$ a su derecha y $10 \mathrm{~cm}$ de altura.

COOPERACIÓN: Los alumnos deberán formar un grupo y repartir las tareas necesarias para la resolución del problema pudiendo elegir cualquiera de las metodologías de Diseño explicadas en clase: Ingeniería Secuencial (un sector del grupo ejecuta las tareas que le corresponde, transfiere los resultados al siguiente y queda a la espera de una segunda interacción) o Ingeniería Concurrente (todos los sectores del grupo desarrollan las tareas coordinadamente y paralelamente de manera que todas las actividades ocurran al mismo tiempo y con decisiones consensuadas).

ENTREGABLES: Se deberá entregar unos informes que indiquen los resultados obtenidos en todas las fases que implica un DDP: estudio de la técnica, planificación del trabajo, diseño conceptual y de detalle, cálculo, documentación de taller, fabricación, ensamblaje y puesta en marcha. Al final de la práctica, los alumnos se expondrá una presentación con los resultados obtenidos en las diferentes fases y se evaluará por los profesores.

ANÁLISIS DE LA PRÁCTICA: Los alumnos expondrán una matriz DAFO para obtener las principales debilidades, amenazas, fortalezas y oportunidades que haya ofrecido esta práctica. Los profesores que imparten la asignatura realizarán una evaluación de los principales indicadores del éxito sobre la mejora de la calidad en la docencia, indicando un

(cc) EY-Nc-ND 2018, Universitat Politècnica de València

Congreso IN-RED (2018) 
valor numérico: mucho (4), bastante (3), poco (2) o nada (1), para evaluar la incidencia del proyecto en el programa docente.

\section{Resultados obtenidos y discusión}

Los alumnos eligieron trabajar según la metodología de Ingeniería Secuencial, esto es, se reparten las tareas entre ellos, según las fases a entregar, y realizan varias iteraciones, pero sin interactuar entre ambos. La elección de este modelo vino motivada por sus preferencias de trabajar de manera individual y gestionando sus propios tiempos. Los resultados de las diferentes fases se muestran en la Tabla 1.

Tabla 1. Cuadro resumen de las actividades realizadas en la práctica, los resultados obtenidos, las decisiones tomadas y las principales herramientas usadas.

\begin{tabular}{|c|c|c|c|c|}
\hline & Actividades & Resultados & Decisión & Herramientas \\
\hline 1 & $\begin{array}{l}\text {-Búsqueda de } \\
\text { información de } \\
\text { máquinas similares } \\
\text {-Lluvia de ideas }\end{array}$ & $\begin{array}{l}\text {-Listado de máquinas } \\
\text { similares y webs de } \\
\text { consulta } \\
\text {-Listado de ideas } \\
\text { sobre mecanismos, } \\
\text { materiales }\end{array}$ & $\begin{array}{l}\text {-Mecanismo pantógrafo para } \\
\text { mantener la horizontalidad de } \\
\text { una pinza de apertura en } \\
\text { movimientos de elevación. } \\
\text {-Actuadores hidráulicos con } \\
\text { cilindros (jeringuillas) para } \\
\text { los diferentes movimientos. } \\
\text {-Material: madera (ligero y de } \\
\text { fácil manipulación). }\end{array}$ & $\begin{array}{l}\text {-Base de datos: } \\
\text { Google y } \\
\text { propias de la } \\
\text { biblioteca UCO. }\end{array}$ \\
\hline 2 & $\begin{array}{l}\text {-Planificación del } \\
\text { trabajo y } \\
\text { estimación de } \\
\text { costes para } \\
\text { abastecimiento de } \\
\text { recursos. }\end{array}$ & $\begin{array}{l}\text {-Cronograma } \\
\text { (diagrama de Gantt) } \\
\text { de actividades. } \\
\text {-Presupuesto de } \\
\text { medios y materiales } \\
\text { necesarios }\end{array}$ & $\begin{array}{l}\text {-Cada equipo realiza una } \\
\text { actividad ( } 1 \text { semana) y pasa } \\
\text { los resultados al siguiente con } \\
\text { interacción mínima. } \\
\text {-50 € de materiales (madera, } \\
\text { pegamentos y tornillería). }\end{array}$ & $\begin{array}{l}\text {-Project } \\
\text { (Microsoft): } \\
\text { Cronogramas. } \\
\text {-Excel } \\
\text { (Microsoft): } \\
\text { Presupuesto. }\end{array}$ \\
\hline 3 & $\begin{array}{l}\text {-Bocetos del } \\
\text { mecanismo. } \\
\text {-Diseño de detalle } \\
\text { con un modelo } \\
\text { CAD }\end{array}$ & & $\begin{array}{l}\text {-Utilizar el diseño CAD } \\
\text { planteado para la fabricación } \\
\text { de la maqueta. } \\
\text { Modificaciones en una } \\
\text { segunda iteración para } \\
\text { incorporar los actuadores }\end{array}$ & $\begin{array}{l}\text {-SolidWorks } \\
2010 \text { (Dassault } \\
\text { Systemes): } \\
\text { Diseño } \\
\text { paramétrico y } \\
\text { ensamblaje. }\end{array}$ \\
\hline 4 & $\begin{array}{l}\text {-Cálculo de } \\
\text { tensiones. } \\
\text {-Cálculo } \\
\text { cinemático de } \\
\text { recorridos. } \\
\text {-Cálculo de } \\
\text { sistema hidráulico. }\end{array}$ & & $\begin{array}{l}\text {-Redefinir ligeramente el } \\
\text { diseño planteado. } \\
\text {-Integración de jeringuillas en } \\
\text { los alojamientos del diseño. }\end{array}$ & $\begin{array}{l}\text {-SolidWorks } \\
\text { 2010: Cálculo } \\
\text { estático. } \\
\text {-Winmecc } \\
\text { (UMA): } \\
\text { Cinemática de } \\
\text { mecanismos. } \\
\text {-Modelos } \\
\text { cinemáticos. }\end{array}$ \\
\hline
\end{tabular}




\begin{tabular}{l|l|l|l|l|}
\hline 5 & $\begin{array}{l}\text {-Generación de } \\
\text { documentación } \\
\text { para la fabricación } \\
\text { de la maqueta. }\end{array}$ & $\begin{array}{l}\text {-Planos técnicos de los } \\
\text { componentes a montar } \\
\text {-Órdenes de trabajo y } \\
\text { planificación de procesos a } \\
\text { realizar. } \\
\text {-Corte de piezas. Algunas de } \\
\text { ellas fueron obtenidas } \\
\text { mediante una fresadora 2D. }\end{array}$ & $\begin{array}{l}\text {-SolidWorks } \\
\text { 2010: Planos } \\
\text {-Vetric Aspire): } \\
\text { CNC para corte. } \\
\text {-Máquinas } \\
\text { CNC. } \\
\text {-Herramientas } \\
\text { de bricolaje. }\end{array}$ \\
\hline 6 & $\begin{array}{l}\text {-Automatización } \\
\text { de movimientos } \\
\text { con elementos de } \\
\text { control. }\end{array}$ & $\begin{array}{l}\text {-Propuesta práctica en } \\
\text { la que los } \\
\text { movimientos (base de } \\
\text { giro) se realizarían } \\
\text { con servomotores } \\
\text { controlados por una } \\
\text { placa de Arduino. }\end{array}$ & $\begin{array}{l}\text {-Debido a la falta de } \\
\text { entendimiento y mala gestión } \\
\text { nel tiempo no se implantó }\end{array}$ & $\begin{array}{l}\text {-Arduino 1.8.5 } \\
\text { (IDE). }\end{array}$ \\
\hline 7 & $\begin{array}{l}\text {-Ensamblaje y } \\
\text { puesta a punto de } \\
\text { componentes }\end{array}$ & &
\end{tabular}

La matriz DAFO de los alumnos sobre el ejercicio planteado se muestra en la Tabla 2.

Tabla 2. Matriz DAFO sobre el ejercicio planteado

\begin{tabular}{lll}
\hline \multirow{3}{*}{ Debilidades } & o & Acabado de las piezas. \\
& o & Fases de trabajo secuenciales. \\
& o & Carencia de conocimientos base (física, matemáticas, etc.). \\
& o & Cambio de mentalidad en la enseñanza-evaluación. \\
\hline \multirow{4}{*}{ Amenazas } & o & Subestimar el proyecto. \\
& o & Tiempo limitado en clase. \\
& 0 & Necesidad de conocimiento de software. \\
& 0 & Falta de comunicación entre equpos. \\
\hline \multirow{4}{*}{ Fortalezas } & 0 & Acceso a todo tipo de información. \\
& 0 & Asesoramiento del profesorado. \\
& 0 & Disponibilidad de máquinas, herramientas y software \\
& 0 & Gran flexibilidad para la creativdad de la solución. \\
\hline \multirow{3}{*}{ Oportunidades } & 0 & Comprender de manera práctica los mecanismos. \\
& 0 & Poner en marcha un proyecto real. \\
& 0 & Comprender pros y cons de ingeniería concurrente vs secuencial. \\
& 0 & Aprender sofware específico que no se cursa en la titulación. \\
\hline
\end{tabular}

(cc) EY-NC-ND 2018, Universitat Politècnica de València

Congreso IN-RED (2018) 
Los resultados de mejora en los indicadores de mejora docente, con la introducción de este proyecto, según el profesorado, indican que el uso de las tutorías, la asistencia a clase y el interés por la comprensión de los contenidos ha aumentado, pero poco. Además, se ha mejorado la comunicación entre los alumnos, aunque finalmente eligieran el modo trabajo de menor cooperación. Todo ello, se ha plasmado en una mejora del rendimiento de los alumnos en sus notas finales.

La comunicación entre el alumno y el profesor ha aumentado poco, pero ha mejorado bastante el clima de trabajo y la participación en clase del alumnado. Ha mejorado bastante la coordinación de actividades entre el profesorado a la hora de la planificación de la asignatura, por la necesidad de adaptarla levemente y dotarla con un enfoque más práctico. Ello ha permitido que el grado de cumplimiento de los objetivos de la asignatura sea bastante mejor al de otros años. La realización del proyecto ha permitido trabajar las siguientes competencias de la titulación: CB2 (resolución de problemas con creatividad, iniciativa, metodología y razonamiento crítico); CB4 (búsqueda y utilización de la normativa y reglamentación relativa a su ámbito de actuación); CU2 (conocer y perfeccionar el nivel de usuario en el ámbito de las TIC); CEC7 (capacidad para conocer, comprender y utilizar los principios de ingeniería del medio rural: estructuras, construcción, hidráulica, máquinas, proyectos técnicos); CEC9 (toma de decisiones mediante el uso de los recursos disponibles para el trabajo en grupos multidisciplinares); CEMC4 (conocer, comprender y utilizar los principios de mecanización agraria).

\section{Conclusiones}

El ejercicio propuesto como un proyecto ha cubierto de manera práctica las competencias a adquirir en la asignatura, permitiendo descubrir otros recursos como software específico ingenieril. La elección de la metodología de trabajo secuencial ha permitido que los alumnos comprendan las desventajas y utilidades de esta frente a una concurrente, pero la elección de esta última lo posibilitaría del mismo modo. Sin embargo, ello ha conllevado a que la resolución técnica de la práctica no haya sido excelente y a que algunas fases, como la automatización, no se haya realizado por falta de coordinación

A nivel docente se ha mejorado la participación e interacción de los alumnos con el profesorado, así como la coordinación horizontal de la asignatura. Las limitaciones de tiempo y recursos pueden ser un escollo para llevar a cabo el proyecto con un grado de éxito mayor, aunque los alumnos admiten que es una oportunidad para utilizar nuevos medios que no podrían aprender con la enseñanza tradicional. La integración vertical de esta práctica con asignaturas afines permitiría aumentar el tiempo para desarrollar contenidos complementarios como software, aunque si no es así, los alumnos podrían disfrutar de cursos de formación permanente que complementen sus competencias.

\section{Agradecimientos}

Los autores del trabajo agradecen a la Universidad de Córdoba la financiación recibida en proyectos de innovación docente. 


\section{Referencias}

GARCÍA, A. E. (2015). "El aprendizaje por proyectos y el trabajo colaborativo, como herramientas de aprendizaje, en la construcción del proceso educativo, de la Unidad de aprendizaje” en RIDE Revista Iberoamericana para la Investigación y el Desarrollo Educativo, 3(5), 123-138.

FOSNOT, C. T. \& Perry, R. S. (1996). “Constructivism: A Psychological theory of Learning”, en Constructivism: Theory, Perspective and Practice, Teacher College, 2, 8-33

MOURSUND, D. G. (1999). "Project-based learning using information technology” en International society for technology in education.

MILLS, J. E., y TREAGUST, D. F. (2003). “Engineering education-Is problem-based or projectbased learning the answer" en Australasian journal of engineering education, 3(2), 2-16.

HAN, S., y BHATTACHARYA, K. (2001). “Constructionism, learning by design, and project based learning” en Emerging perspectives on learning, teaching, and technology. E-book (http://www.coe.uga.edu/epltt/LearningbyDesign.htm . [Consulta: 15 de Marzo de 2018]

HMELO-SILVER, C. E. (2004). “Problem-based learning: What and how do students learn?” en Educational psychology review, 16(3), 235-266

PARRA-COSTA, C. J., et al. (2011). "Estrategias de coordinación horizontal y vertical en los planes de estudios adaptados al EEES” en Congreso Internacional de Innovación Docente, Cartagena, 2011. Cartagena: Universidad Politécnica de Cartagena, 2011. p.1815-1824

(cc) EY-NC-ND 2018, Universitat Politècnica de València 